\title{
Resonant second-harmonic generation in monosized and aligned single-walled carbon nanotubes
}

\author{
Hui Min Su, Jian Ting Ye, Zi Kang Tang, and Kam Sing Wong* \\ Department of Physics, Hong Kong University of Science and Technology, Kowloon, Hong Kong, People's Republic of China
}

(Received 16 January 2008; published 26 March 2008)

\begin{abstract}
The magnitude and spectral response of second-harmonic generation (SHG) from $0.4 \mathrm{~nm}$ single-walled carbon nanotubes (CNTs) in the channels of $\mathrm{AlPO}_{4}-5$ zeolite is reported. The second harmonics (SH) was found to be polarized perpendicular to the tube axis and maximized by an excitation polarization at $45^{\circ}$ to the tube axis. A SH resonance peak at $2 \mathrm{eV}$ was observed, which corresponds to the lowest-energy excitonic state in chiral $(4,2)$ CNTs. The second-order optical susceptibility $\chi^{(2)}$ of the system was determined to be $10^{-6}$ esu, which agrees with the large $\chi^{(2)}$ predicted for small diameter CNTs. These experimental results suggest that SHG can be used to characterize the symmetry and chirality of CNTs.
\end{abstract}

DOI: $10.1103 /$ PhysRevB.77.125428

PACS number(s): 42.65.An, 73.22.-f, 73.63.Fg

\section{INTRODUCTION}

The nonlinear optics of carbon nanotubes (CNTs) is a subject of increasing interest. Intensive theoretical ${ }^{1,2}$ and experimental ${ }^{3}$ investigations have shown that CNTs exhibit large and fast electronic optical nonlinearity because of their quasi-one-dimensional structure. This suggests the possibility of applications in optical harmonics generation and ultrafast optics and photonics.

Many of the unusual electronic and optical properties of CNTs are due to their ultrasmall diameter and large curvature. However, these same properties also entail substantial challenges in characterizing specific CNTs. Direct structural analysis techniques such as $\mathrm{x}$ ray and transmission electron microscopy must operate near the margin of their sensitivity. On the other hand, the optical properties of CNTs depend sensitively on their atomic structure and chirality, ${ }^{1}$ so their experimentally measured optical properties can be taken as indirect, yet convincing evidence of their behavior and used to verify theoretical predictions.

In particular, nonlinear optical (NLO) processes obey selection rules distinct from those governing linear optical processes and thus can provide complementary insights into the electronic structure of these ultrasmall CNTs. Currently, little research activity has addressed CNTs' second-harmonic generating abilities because the second-order nonlinear susceptibility $\chi^{(2)}$ vanishes for centrosymmetric materials and racemic mixtures. In CNT materials, the optical second harmonics (SH) can be generated through surface interactions, local imperfections, deformation, or the macroscopic chirality resulting from the nonracemic assembly of the CNT structure. $^{4,5}$ Only those arising from chirality display the spectral feature characteristic of different CNT species. Second-harmonic generation (SHG) signals from chiral CNTs have been experimentally observed in bulk samples and thin films ${ }^{4,5}$ in mixtures of CNT topologies and orientations, demonstrating the second-harmonic generating abilities of CNTs, whereas the anisotropy information in the SHG process may be lost in these samples. In this paper, SHG in monosized $(0.4 \mathrm{~nm})$ and well-aligned single-walled carbon nanotubes (SWCNTs) is studied. Unlike earlier SHG experiments, studying SWCNTs in which the SHG was mainly due to surface anisotropies or local deformations in the structure, ${ }^{4,5}$ the SHG signals observed in our experiments were associated with the CNTs' chirality and hence to be compared directly with theoretical calculations ${ }^{1}$ of $\chi^{(2)}$ for chiral carbon nanotubes. The totally parallel alignment of the SWCNTs in the sample enabled investigation of the SH's polarization and its dependence on the excitation polarizations. Furthermore, the magnitude and spectrum of $\chi^{(2)}$ of the sample were measured, and it showed an excitation resonance peak at $2.01 \mathrm{eV}$ associated with the $(4,2)$ chiral CNTs.

\section{SAMPLES AND SECOND-HARMONIC GENERATION EXPERIMENTS}

The SWCNTs were grown by pyrolysis of tripropylamine $\left[\left(\mathrm{CH}_{3} \mathrm{CH}_{2} \mathrm{CH}_{2}\right)_{3} \mathrm{~N}\right.$, TPA $]$ in the channels of the host single crystalline aluminophosphate $\mathrm{AlPO}_{4}-5$ (AFI) zeolite. ${ }^{6}$ The AFI crystals containing SWCNTs were typically of about $50 \mu \mathrm{m}$ in diameter and about $300 \mu \mathrm{m}$ long. High-resolution trasmission electron microscopy and Raman scattering measurements showed that the SWCNTs are $0.4 \mathrm{~nm}$ in diameter and aligned parallel to the AFI zeolite's crystal axis. ${ }^{6,7}$ There are three possible structures in the sample: zigzag $(5,0)$, armchair $(3,3)$, and chiral $(4,2)$. Among them, only chiral $(4,2)$ tubes are not centrosymmetric and exhibit second-order NLO behavior. Machón et al. ${ }^{8}$ compared the calculated optical absorption under parallel polarized light for such material with experimental data and estimated the nanotube filling fraction as $2 \times 10^{-4}$ and the concentration of $(5,0),(3,3)$, and $(4,2)$ as 0.5:0.4:0.1.

The SHG process is determined by a second-order nonlinear polarization of the form

$$
\mathbf{P}^{(2)}(2 \omega)=\varepsilon_{0} \chi^{(2)}(-2 \omega: \omega, \omega): \mathbf{E}(\omega) \mathbf{E}^{*}(\omega) .
$$

Because of the one-dimensional chiral symmetry, only two tensor elements of $\chi^{(2)}$ may possibly be nonzero for chiral $(4,2)$ nanotubes: $\chi_{x y z}^{(2)}$ and $\chi_{y z x}^{(2)}\left(z\right.$ along the nanotube axis). ${ }^{1}$ Furthermore, $\chi_{x y z}^{(2)}=-\chi_{y z x}^{(2)}$. As a consequence, the chiralityrelated component of $\mathbf{P}^{(2)}(2 \omega)$ is proportional to ${ }^{9}$

$$
P_{\text {eff }}^{o}(2 \omega) \propto 2 \varepsilon_{0} \chi_{x y z}^{(2)} \sin \beta \cos \beta\left[\mathbf{E}^{e}(\omega)\right]^{2},
$$

where the superscript $o(e)$ denotes the ordinary wave (extraordinary wave) polarized perpendicular (parallel) to the 


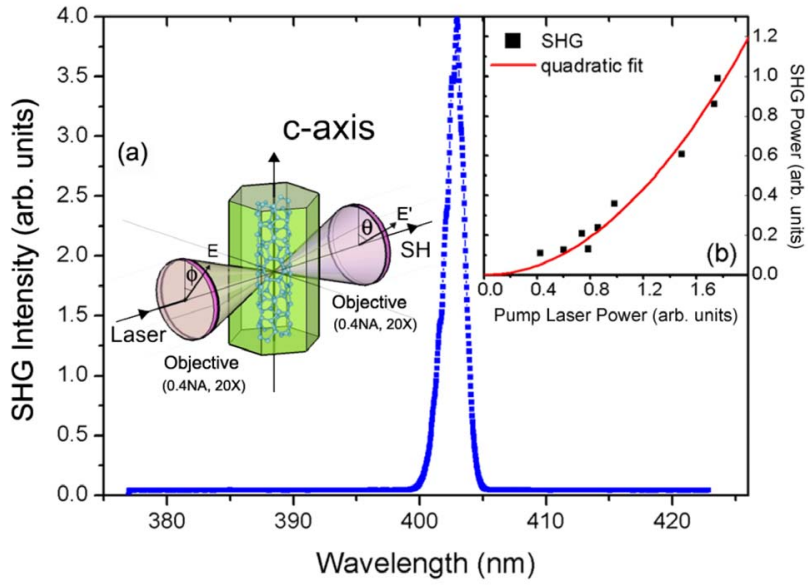

FIG. 1. (Color online) The SHG signal excited by $150 \mathrm{fs}$ light psulses at $800 \mathrm{~nm}$. Inset (a): diagram of the geometry and the polarization arrangement in the experimental setup. Inset (b): the integrated SHG intensity as a function of the pump laser power and the quadratic fit.

principal surface and $\beta$ is the angle of incidence. Therefore, considering a strongly focused laser beam with the beam axis normal to the aligned $(4,2)$ tubes, the total polarization is proportional to

$$
P_{e f f}^{o}(2 \omega) \propto 2 \varepsilon_{0} \chi_{x y z}^{(2)} \iint \sin \beta \cos \beta[\mathbf{E}(\omega, \phi, \Omega)]^{2} d \Omega,
$$

where $\phi$ is the polarization angle of the excitation beam against the nanotube axis and $\Omega$ is the solid angle within the cone of the laser. The integration boundary of $\Omega$ is defined by the numerical aperture of the focusing objective.

Figure 1 shows the $\mathrm{SH}$ radiation from a SWCNT sample excited by $150 \mathrm{fs}$ light pulses at $800 \mathrm{~nm}$ from a Ti:sapphire regenerative amplifier. The dependence of the SHG signal's intensity on the laser energy was measured by varying the excitation power from 0.4 to $1.8 \mathrm{~mW}$, and the result is plotted in Fig. 1(b) together with a quadratic fit. The quadratic dependence of SHG intensity on excitation energy indicates that SHG in the CNTs under investigation is the result of a second-order nonlinear process.

In order to observe the polarization dependence of SHG intensity on the excitation laser, the polarization angle $\phi$ of the laser [as defined in Fig. 1(a)] was changed using a halfwave plate and the polarization of the $\mathrm{SH}$ was tested with an output polarizer. Figure 2(a) shows the intensity of SHG from a bare AFI crystal and the CNT sample (with no discrimination to the SHG polarization) when the excitation laser's polarization angle $\phi$ varied from $-90^{\circ}$ (transverse excitation) to $+90^{\circ}$. SH from a bare AFI crystal was found to be completely polarized along the crystal axis and maximized with excitation parallel to the AFI crystal axis. On the contrary, the integrated $\mathrm{SH}$ radiation from the CNT sample was at maximum when the polarization of the excitation laser was at $45^{\circ}$ to the nanotube axis, and it decreased monotonically by over ten times to a minimum when the excitation

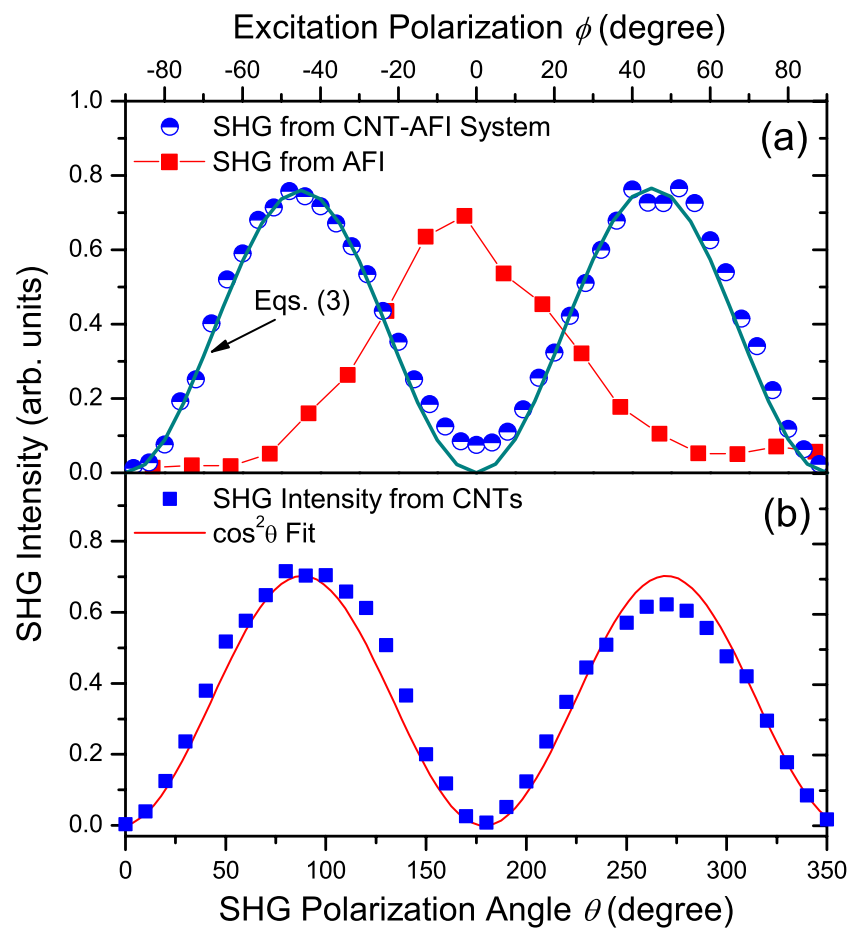

FIG. 2. (Color online) (a) The integrated SHG intensity from SWCNT and from AFI zeolite as a function of the excitation polarization angle $\phi$. The curve pointed by an arrow was calculated using Eqs. (3). (b) The integrated SHG intensity from SWCNT as a function of the emission light polarization angle $\theta$ with the excitation angle $\phi=45^{\circ}$ together with a $\cos ^{2} \theta$ fit.

polarization was parallel or perpendicular to the tube axis. This result fitted nicely the profile calculated using Eqs. (3) as expected [Fig. 2(a)]. In the optimal case, where the excitation polarization was at $45^{\circ}$ to the tube axis, the integrated $\mathrm{SH}$ radiation followed a $\cos ^{2} \theta$ law [Fig. 2(b)], which is consistent with the one-dimensional symmetry of chiral $(4,2)$ CNTs, i.e., $\chi_{x y z}^{(2)}=-\chi_{y z x}^{(2)}$. This confirms that the SHG from the SWCNT sample was mainly linearly polarized in the $x-y$ plane and perpendicular to the tube axis, which agrees with the theoretical prediction for chiral $(4,2)$ tubes. Hence, the possibility of defect-induced SHG can be excluded.

For a given CNT chiral topology, the left-handed $(L)$ and right-handed $(R)$ enantiomers have opposite SHG contributions as determined by their symmetry properties. ${ }^{4}$ It follows that a macroscopic chirality must have existed in the sample, either from a breaking of the spatial symmetry of the CNTs or from an imbalance between the two enantiomers. In our case, there were several mechanisms which may have broken the spatial symmetry of the sample. AFI crystals contain aluminum-oxygen-phosphorus pairs pointing in the same direction along the crystal axis, forming a macroscopically preferred direction. ${ }^{10}$ Hence, an excess of one type of enantiomers may be achieved during the growth process of chiral CNTs because of this asymmetry. Indeed, molecular mechanics computations ${ }^{11}$ demonstrate that for some organic molecules, one helical arrangement is more stable than the other in the channel of AFI crystal, and this can lead to a longrange chirality in the whole solid. Observation of large SHG 
signal strongly indicates that there is an imbalance between the two enantiomers for CNTs grown in the AFI channels. Moreover, the finite length of CNTs with different configurations of hydrogen terminated dangling bonds at each $\mathrm{end}^{7}$ will break the translational symmetry of the tubes which can also contributes to SHG, although this should only play a minor role. In addition, only 100 or AFI channels in the focused beam may contain $(4,2)$ CNTs from which the SH signal is coherent. Consequently, the local imbalance between the two enantiomers will also be reflected in the SH signal.

\section{SPECTRAL RESPONSE OF $\chi^{(2)}$}

According to the polarization anisotropy discussed above, resonant SHG from SWCNT samples was measured with excitation energy tunable from 1.7 to $2.3 \mathrm{eV}$ using the signal output branch of an optical parametric amplifier. ${ }^{12}$ In the case of SHG induced by a focused Gaussian laser beam in a uniaxial nonlinear crystal with strong linear absorption under the resonance conditions, the harmonic field outside the crystal is ${ }^{13}$

$$
\begin{aligned}
\mathbf{E}_{2}(2 \omega ; x, y, z)= & \left(\frac{4 i \pi \omega \mathbf{P}_{\text {eff }}(-2 \omega: \omega, \omega)}{c n_{2 \omega}(1+i \tau)}\right) \\
& \times \exp \left(-\frac{1}{2} \alpha_{2} l+2 i k_{1} z\right) \\
& \times \int_{0}^{l} d z^{\prime} \frac{\exp \left(-\alpha z^{\prime}+i \Delta k z^{\prime}\right)}{1+i \tau^{\prime}} \\
& \times \exp \left(-\frac{2\left\{\left[x-\rho\left(l-z^{\prime}\right)\right]^{2}+y^{2}\right\}}{w_{0}^{2}(1+i \tau)}\right),
\end{aligned}
$$

where the laser beam is focused at $z=f$ with beam waist $w_{0}$ and confocal parameter $b$ in the crystal. $\alpha=\alpha_{1}-\alpha_{2} / 2$, where $\alpha_{1}$ and $\alpha_{2}$ are the absorption coefficients for the fundamental and the harmonic fields. $l$ is the thickness of the crystal, $\rho$ is the double refraction angle, $\Delta k$ is the mismatch wave vector, and $\tau^{\prime}=2\left(z^{\prime}-f\right) / b$. When $\Delta k$ is ignored and $\rho$ is assumed to be constant over the frequency range being investigated, the SHG intensity $I(2 \omega)$ can be written as

$$
\frac{I(2 \omega)}{I^{2}(\omega)} \propto \omega^{2}\left|\chi_{e f f}^{(2)}(-2 \omega: \omega, \omega)\right|^{2} F_{a b s}(\omega)\left|\int_{-1}^{1} d t \frac{\left[F_{a b s}(\omega)\right]^{t / 2}}{1+i t^{2}}\right|^{2},
$$

where $F_{a b s}(\omega)=\exp [-\alpha(\omega) l-\alpha(2 \omega) l / 2]$. Because the absorption spectrum varied slowly within the energy range due to the low nanotube concentration in the sample, ${ }^{8}$ the efficiency of SHG was mainly determined by the $\left|\chi_{\text {eff }}^{(2)}\right|^{2}$ of the $(4,2)$ tubes. Figure 3 shows the measured intensity of SHG against the excitation frequency from 1.7 to $2.3 \mathrm{eV}$. The solid red line is the computation of SHG efficiency based on Eq. (5) using the $\chi^{(2)}$ of chiral $(4,2)$ tubes calculated by Guo et al. ${ }^{1}$ and the linear optical absorption data of Li et al. ${ }^{7}$

A sharp, asymmetric resonance peak elongated toward the higher energies is observed in the SHG spectrum. The

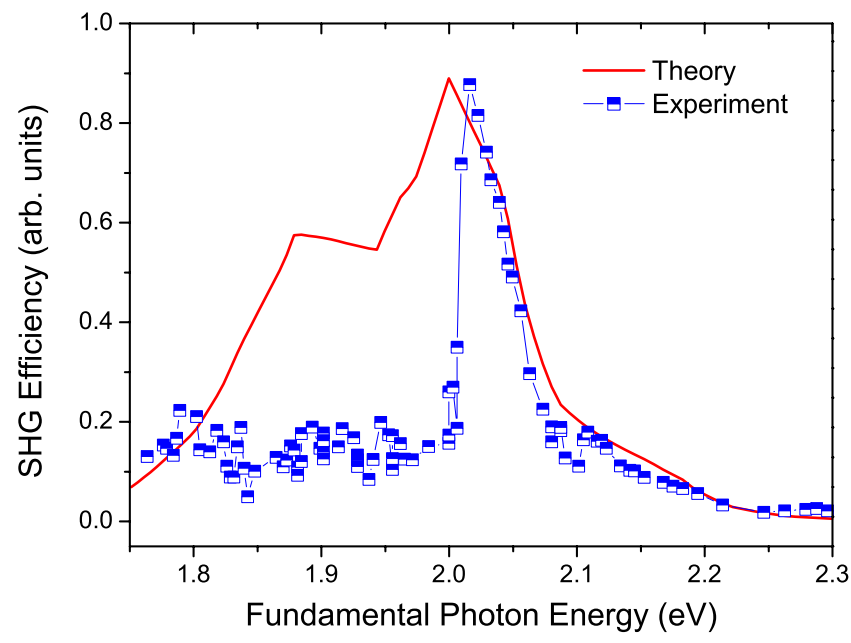

FIG. 3. (Color online) SHG intensity as a function of the excitation laser wavelength and the theoretical curve for chiral $(4,2)$ tubes. The absorption of fundamentals and second harmonics through the sample used in the calculation was taken from Ref. 7.

experimental SHG spectrum agrees with the theoretical curve very well in the higher-energy region as well as the peak position at $2.01 \mathrm{eV}$, but the resonance peak appears to be much narrower. This sharp feature observed in the resonant SHG spectrum suggests the presence of strongly bound excitons in the system, which were neglected in the calculated curve. The calculation of $\chi^{(2)}$ in Ref. 1 was based on the independent-particle approximation, where excitonic effects and local-field corrections were not included. The peak at $2.0 \mathrm{eV}$ was then suggested to have arisen from the intense contribution of the fundamental band edge transition $\left(h \omega \sim E_{g} \sim 2.0 \mathrm{eV}\right)$ in $(4,2)$ tubes. However, it has become clear that electron-electron interactions play an important role in CNT systems due to the confinement of the electronic states. ${ }^{14}$ For $(4,2)$ tubes, the lowest bright excitonic state $\left(E_{11}^{1 u}\right)$ is calculated to be $2.2 \mathrm{eV}$ above the valence band edge with a binding energy of $0.9 \mathrm{eV} .{ }^{15}$ There will be $0.1-0.2 \mathrm{eV}$ redshift of the transition energy for a relaxed tube structure compared to the cylindrical, unrelaxed model according to the calculations of Machón et al. ${ }^{8}$ Moreover, another 50-60 meV decrease would be expected for CNTs in an AFI crystal due to the external dielectric screening. Considering the real situation in the sample, the agreement on the exciton transition energy between the experimental and calculated results is satisfactory. The resonant peak at $2.01 \mathrm{eV}$ in the observed SH excitation spectrum therefore corresponds to the transition to the lowest optically active exciton state, $E_{11}^{1 u}$. Consequently, the high-energy tail of the peak is attributed to exciton-phonon interaction. ${ }^{16,17}$ The coupling between the bright exciton and the vibration of the host matrix can lead to the localization of the exciton wave function in real space and the mixture of excitonic states in $K$ space. Part of the oscillator strength is transferred to the dark excitons that lie close to the bright exciton at the bottom of the band, resulting in a high-energy tail and a slight redshift in the spectrum. 


\section{ESTIMATION OF $\chi^{(2)}$ MAGNITUDE}

To estimate the order of magnitude of the $\chi^{(2)}$ coefficient of chiral $(4,2)$ tubes quantitatively, the SHG data were compared with the signal from single-crystal quartz using the same setup with excitation at $800 \mathrm{~nm}$. The size of the focal spot was about one-tenth of the thickness of the SWCNT sample, and the depth of focus was comparable to the thickness of the sample. The orientation of the single-crystal quartz was adjusted to optimize the SH signal. By measuring the ratio of the $\mathrm{SH}$ signal from the sample to that from the quartz, the $\chi^{(2)}$ value of the CNT sample was determined to be about $6 \%$ of that of the quartz. Taking into account the chiral (4,2) tubes' concentration of $2 \times 10^{-5}\left(2 \times 10^{-4} \times 10 \%\right)$ in the sample while the maximum nanotube concentration in the AFI channels was expected to be $8 \%,{ }^{8}$ the second-order susceptibility tensor elements of the AFI-CNT $(4,2)$ sample could be about 240 times $\left[\left(6 \% / 2 \times 10^{-5}\right)\right] \times 8 \%=240$ that of the quartz, or $1.8 \times 10^{-7} \mathrm{esu}$, assuming that all the AFI channels were completely filled with $(4,2)$ tubes. Compared with the SHG spectrum shown in Fig. 3, the $\chi^{(2)}$ of AFI-CNT $(4,2)$ sample was expected to be $2.1 \times 10^{-6}$ esu at the resonance (around $2.0 \mathrm{eV}$ ). This is consistent with the first-principles calculation of $\chi^{(2)}=10^{-5}$ esu for $(4,2)$ tubes, ${ }^{1}$ considering that the maximum volume of nanotube was only $8 \%$ in the zeolite channels.

\section{CONCLUSIONS}

In summary, we have studied the polarization dependence of the second-harmonics generated from monosized and well-aligned SWCNTs in the channels of AFI zeolite. The second harmonics from chiral $(4,2)$ tubes is mainly polarized perpendicular to the tube axis and maximized when the polarization of the excitation is at $45^{\circ}$ to the tube axis. The resonance peak was observed at $2 \mathrm{eV}$ in the $\mathrm{SHG}$ excitation spectrum. It was attributed to the intense contribution from the lowest bright exciton, $E_{11}^{1 u}$, in $(4,2)$ tubes. The magnitude of the second-order optical susceptibility coefficient $\chi^{(2)}$ of the AFI-CNT $(4,2)$ sample was estimated to be about $2.0 \times 10^{-6}$ esu at the resonance, assuming all channels in the AFI were completely filled with $(4,2)$ tubes. The experimental results are consistent with the theoretical prediction of $\chi^{(2)}$ for chiral carbon nanotubes and suggest that SHG can be used to characterize the symmetry, diameter, and chirality of CNTs.

\section{ACKNOWLEDGMENTS}

This work was supported by the Research Grant Council of Hong Kong (Grants No. 604405 and No. 605003, by RGC Central Allocation No. CA04/05.SC02), and the HKUST President's direct allocation (No. F0204). *phkswong@ust.hk

${ }^{1}$ G. Y. Guo, K. C. Chu, D. S. Wang, and C. G. Duan, Phys. Rev. B 69, 205416 (2004).

${ }^{2}$ G. Y. Slepyan, S. A. Maksimenko, V. P. Kalosha, J. Herrmann, E. E. B. Campbell, and I. V. Hertel, Phys. Rev. A 60, R777 (1999); O. E. Alon, V. Averbukh, and N. Moiseyev, Phys. Rev. Lett. 85, 5218 (2000).

${ }^{3}$ X. Liu, J. H. Si, B. H. Chang, G. Xu, Q. G. Yang, Z. W. Pan, S. S. Xie, P. X. Ye, J. H. Fan, and M. X. Wan, Appl. Phys. Lett. 74, 164 (1999); C. Stanciu, R. Ehlich, V. Petrov, O. Steinkellner, J. Herrmann, I. V. Hertel, G. Ya Slepyan, A. A. Khrutchinski, S. A. Maksimenko, and F. Rotermund, ibid. 81, 4064 (2002); O. J. Korovyanko, C. X. Sheng, Z. V. Vardeny, A. B. Dalton, and R. H. Baughman, Phys. Rev. Lett. 92, 017403 (2004); J. S. Lauret, C. Voisin, G. Cassabois, C. Delalande, P. Roussignol, O. Jost, and L. Capes, ibid. 90, 057404 (2003); Y. Z. Ma, L. Valkunas, S. L. Dexheimer, S. M. Bachilo, and G. R. Fleming, ibid. 94, 157402 (2005); L. Perfetti, T. Kampfrath, F. Schapper, A. Hagen, T. Hertel, C. M. Aguirre, P. Desjardins, R. Martel, C. Frischkorn, and M. Wolf, ibid. 96, 027401 (2006).

${ }^{4}$ L. De Dominicis, S. Botti, L. S. Asilyan, R. Ciardi, R. Fantoni, M. L. Terranova, A. Fiori, S. Orlanducci, and R. Appolloni, Appl. Phys. Lett. 85, 1418 (2004).

${ }^{5}$ D. A. Akimov, M. V. Alfimov, S. O. Konorov, A. A. Ivanov, S. Botti, A. A. Podshivalov, R. Ciardi, L. De Dominicis, L. S. Asilyan, R. Fantoni, and A. M. Zheltikov, J. Exp. Theor. Phys. 98, 220 (2004); S. O. Konorov D. A. Akimov, A. A. Ivanov, M. V. Alfimov, S. Botti, R. Ciardi, L. De Dominicis, and A. M.
Zheltikov, J. Raman Spectrosc. 34, 1018 (2003).

${ }^{6}$ Z. K. Tang, H. D. Sun, J. Wang, J. Chen, and G. Li, Appl. Phys. Lett. 73, 2287 (1998); N. Wang, Z. K. Tang, G. D. Li, and J. S. Chen, Nature (London) 408, 50 (2000); Z. M. Li, H. J. Liu, J. T. Ye, C. T. Chan, and Z. K. Tang, Appl. Phys. A: Mater. Sci. Process. 78, 1121 (2004).

${ }^{7}$ Z. M. Li, Z. K. Tang, H. J. Liu, N. Wang, C. T. chan, R. Saito, S. Okada, G. D. Li, J. S. Chen, N. Nagasawa, and S. Tsuda, Phys. Rev. Lett. 87, 127401 (2001).

${ }^{8}$ M. Machón, S. Reich, C. Thomsen, D. Sanchez-Portal, and P. Ordejon, Phys. Rev. B 66, 155410 (2002).

${ }^{9}$ N. Ji, and Y. R. Shen, Chirality 18, 146 (2006).

${ }^{10}$ G. J. Klap, S. M. van Klooster, M. Wubbenhorst, J. C. Jansen, H. van Bekkum, and J. van Turnhout, J. Phys. Chem. B 102, 9518 (2005).

${ }^{11}$ L. Gomez-Hortiguela, F. Cora, C. R. A. Catlow, and J. PerezPariente, Phys. Chem. Chem. Phys. 8, 486 (2006).

${ }^{12}$ K. S. Wong, Z. R. Qui, H. Wang, and G. K. L. Wong, Opt. Lett. 22, 898 (1997).

${ }^{13}$ G. D. Boyd and D. A. Kleinman, J. Appl. Phys. 39, 3597 (1968).

${ }^{14}$ G. D. Scholes and G. Rumbles, Nat. Mater. 5, 683 (2006).

${ }^{15}$ E. Chang, G. Bussi, A. Ruini, and E. Molinari, Phys. Rev. B 72, 195423 (2005).

${ }^{16}$ F. Plentz, H. B. Ribeiro, A. Jorio, M. S. Strano, and M. A. Pimenta, Phys. Rev. Lett. 95, 247401 (2005).

${ }^{17}$ M. S. Dresselhaus, G. Dresselhaus, R. saito, and A. Jorio, Annu. Rev. Phys. Chem. 58, 719 (2007). 